\title{
IDENTIFICAÇÃO DE ÁREAS PARA RECOMPOSIÇÃO FLORESTAL COM BASE EM PRINCÍPIOS DE ECOLOGIA DE PAISAGEM ${ }^{1}$
}

\author{
Sílvio Frosini de Barros Ferraz ${ }^{2}$ Carlos Alberto Vettorazzi³,4
}

\begin{abstract}
RESUMO - O manejo de áreas florestais com base em princípios de ecologia da paisagem é uma tendência do setor florestal, que tem como proposta o gerenciamento integrado dos aspectos econômicos, sociais e ambientais da atividade produtiva, envolvendo decisões complexas que podem ser auxiliadas pelas técnicas de geoprocessamento. No presente trabalho objetivou-se o desenvolvimento de uma metodologia para aplicação dos conceitos de ecologia de paisagem no planejamento do uso da terra em áreas de reflorestamento, por meio da utilização de um sistema de informações geográficas. Para tal, utilizou-se como área de estudo uma fazenda de reflorestamento da International Paper do Brasil, tendo sido estabelecidos cinco critérios para determinação de áreas para recomposição: fertilidade dos solos, mata nativa existente, corpos d'água, declividade e suscetibilidade à erosão. Esses fatores foram analisados, empregando-se os recursos de decisão multicritérios, em ambiente SIG. Como resultado foi obtido um mapa com áreas adequadas à recomposição florestal, segundo os critérios adotados. Com este mapa realizou-se uma simulação, alocando uma nova área de floresta nativa, e o resultado foi avaliado em nível de paisagem por meio de índices apropriados.
\end{abstract}

Palavras-chave: Fragstats, recomposição florestal, reflorestamento, SIG e suporte à decisão.

\section{IDENTIFICATION OF SUITABLE AREAS FOR FOREST RECOVERY BASED ON PRINCIPLES OF LANDSCAPE ECOLOGY}

\begin{abstract}
Forestland management based on landscape ecology principles is a tendency in the forest sector, whose proposal is the integrated management of economic, social, and environmental factors, taking into account complex decisions supported by geospatial technologies. The objective of this study was to develop a methodology to apply the concepts of landscape ecology in land use planning of forest farms, using Geographic Information System techniques. The study area was a forest farm owned by the International Paper do Brasil. Five criteria were used to identify suitable areas for forest recovery: soil fertility, existing native forest patches, water bodies, slope, and erosion susceptibility. These factors were analyzed through the multi-criteria decision resources in a GIS environment. The result was a map showing suitable areas for forest recovery, according to the defined criteria. Based on this map, a simulation was made to allocate a new forest area and the results were evaluated at a landscape level using proper indices.
\end{abstract}

Key words: Fragstats, forest recovery, forest plantation, GIS and decision support.

\section{INTRODUÇÃO}

Com o avanço tecnológico, a "paisagem natural" vem sendo substituída pela "paisagem urbana" e pela "paisagem rural". As drásticas mudanças na "paisagem natural" têm causado problemas ecológicos e sociais (Magro, 1997). Os problemas ecológicos podem ser avaliados pelas alterações na relação da paisagem com os organismos vivos, que é o objeto de estudo da ecologia de paisagem. A concepção de ecologia de paisagem foi

1 Recebido para publicação em 29.5.2002.

Aceito para publicação em 9.9.2003.

2 Engenheiro Florestal, doutorando em Recursos Florestais pela Escola Superior de Agricultura "Luiz de Queiroz" - ESALQ/ USP, Av. Pádua Dias, 11 - Dep. de Engenharia Rural, 13418-900 Piracicaba-SP; ${ }^{3}$ Dep. de Engenharia Rural - ESALQ/USP;

${ }^{4}$ Autor correspondente. 
inicialmente influenciada por cientistas naturais preocupados com a relação entre o padrão de distribuição de plantas e animais e os meios físico e antrópico (Biogeografia). Mais tarde, engenheiros florestais, agrônomos e arquitetos, preocupados com o planejamento do uso da terra, interessaram-se pela ecologia de paisagem. O conceito "manejo de paisagem" surgiu da aplicação dos conceitos da ecologia de paisagem ao manejo de ecossistemas naturais (Viana \& Oliveira, 1997).

$\mathrm{O}$ conceito de manejo de paisagem em áreas florestais pode ser considerado mais abrangente que o manejo florestal tradicional, levando em conta não apenas o aspecto de produção, mas também os aspectos ambiental, social e estético das florestas, entre outros (Boyce \& McNab, 1994). A discussão do conceito de manejo de paisagens é recente no setor florestal brasileiro e tem sido estimulada pelo processo de definição dos princípios e critérios estabelecidos pelo Conselho para o Manejo Florestal (FSC), que garantem o "bom manejo florestal", fundamental para certificação de produtos florestais (Viana \& Oliveira, 1997).

A aplicação do conceito de manejo de paisagens tem como objetivos principais a formação de florestas produtivas, mas com menores riscos ao ambiente; a manutenção da biodiversidade; a maior integração; e a utilização social, proporcionando uma paisagem visualmente agradável. Para aplicação prática desses conceitos de manejo deve-se considerar que, como uma proposta holística e integrada de manejar recursos, o manejo de paisagens envolve decisões com base em complexas interações de fatores bióticos e abióticos (Lachowski et al., 1994). As facilidades que o sensoriamento remoto (SR) e os sistemas de informações geográficas (SIG) oferecem para o processamento e a análise de dados espaciais tornam estas técnicas fundamentais para análise, monitoramento e modelagem das práticas de manejo (Lachowski et al., 1994; Oliver, 1992).

Vários trabalhos foram realizados com o SIG, para auxiliar o manejo de áreas agrícolas e naturais. Formaggio et al. (1992), utilizando informações como declividade, solo e uso da terra, e empregando o sistema SGI/INPE, produziram um mapa de aptidão agrícola, que é um poderoso instrumento de planejamento do uso da terra. $\mathrm{O}$ mapeamento de risco potencial de erosão foi realizado para uma microbacia por Ridente Júnior et al. (1997), utilizando o cruzamento entre mapas de solos e declividade. Bergen et al. (1995) utilizaram o SIG para prever o impacto visual decorrente das operações de corte raso em florestas por meio do modelo digital do terreno e o mapa de estradas.

Zhou \& Civco (1996) classificaram o processo de decisão auxiliado pelo SIG em dois procedimentos: sobreposição de mapas temáticos e análise de decisão multicriterial. O método de sobreposição foi utilizado por Ferraz \& Vettorazzi (1998), que associaram vários fatores espaciais para determinação das principais áreas de risco de incêndios florestais, auxiliando a decisão de alocação de recursos e manejo diferenciado na área de estudo.

Segundo Eastman et al. (1995), o processo de aplicação de uma regra de decisão é chamado de avaliação. Para atingir um objetivo específico, é comum avaliar vários critérios ou fatores. Este procedimento é chamado de avaliação multicriterial ou análise de decisão multicriterial. Alguns autores utilizaram a técnica de avaliação multicriterial para estudos ambientais. Lathrop \& Bognar (1998) utilizaram esta técnica para escolha de áreas prioritárias para conservação na Sterling Forest, em New Jersey, Estados Unidos. Vettorazzi et al. (2000) empregaram a abordagem multicritérios/único objetivo, em ambiente SIG, para mapear risco de incêndios florestais em uma bacia hidrográfica, no Estado de São Paulo.

O objetivo do presente trabalho foi o desenvolvimento de uma metodologia para auxiliar na identificação de áreas mais adequadas à recomposição florestal em áreas de reflorestamento, utilizando princípios de ecologia da paisagem.

\section{MATERIAL E MÉTODOS}

A área utilizada para o estudo foi a fazenda "Santa Fé B", localizada no município de Brotas-SP, de propriedade da empresa International Paper do Brasil, cuja finalidade é produção de madeira de eucalipto para fabricação de celulose e papel. A precipitação média anual na região é de $1.250 \mathrm{~mm}$ e a temperatura média anual é de $21^{\circ} \mathrm{C}$. O relevo é ondulado, predominando solos do tipo Latossolo Vermelho-Amarelo, fase arenosa (Rodrigues, 1986).

O software de SIG empregado foi o IDRISI 2.0. Para criação de um banco de dados cartográficos com todas as informações empregadas no trabalho, utilizou-se o ArcView 3.0. As seguintes informações foram utilizadas no trabalho: 
Relevo e Hidrografia - A partir das curvas de nível digitalizadas da carta topográfica na escala 1:50.000, do IBGE, utilizou-se o software Surfer 6.0 para interpolação, pelo método da krigagem, gerando-se o modelo digital do terreno (MDT). A rede hidrográfica foi obtida a partir da mesma carta.

Solos - Utilizou-se a carta do Levantamento Pedológico Semidetalhado do Estado de São Paulo, quadrícula de Brotas (Oliveira et al., 1981), na escala de 1:100.000.

Uso da Terra - Todas as informações de uso da terra foram obtidas com base nas unidades produtivas (talhões) e áreas naturais referentes à situação existente em 1998. Relacionando-se o número de cada talhão com o cadastro florestal da empresa, pode-se obter o mapa de uso atual da terra.

A escolha das áreas para recomposição florestal foi baseada em cinco critérios, para os quais foram produzidos os seguintes planos de informação (mapas temáticos):

- Declividade (DECLIV) - o mapa com as declividades, em porcentagem, foi obtido a partir do MDT. Considerou-se que áreas mais declivosas são mais adequadas à recomposição, devido à necessidade de proteção permanente do solo e à dificuldade de mecanização destas áreas para o processo produtivo.

- Proximidade de rios e lagos (PROXRIO) - obtida a partir do mapa de hidrografia, gerando-se um gradiente de proximidade aos corpos d'água. Considerou-se que áreas mais próximas aos corpos d'água são mais adequadas à recomposição, devido ao fato de a água ser essencial à fauna, à necessidade de maior proteção dos recursos hídricos e à ocorrência de atividades de lazer nestes locais.

- Proximidade de mata nativa (PROXNAT) - obtida pelo mesmo procedimento adotado em PROXRIO, mas em relação aos polígonos de vegetação nativa. Considerou-se que áreas próximas à vegetação nativa são mais adequadas à recomposição, pelo fato de aumentar a continuidade da vegetação natural, aumentando assim as possibilidades de troca genética e a área contínua disponível para os movimentos da fauna local.

- Suscetibilidade à erosão (SUSCERO) - obtida por meio de uma adaptação da metodologia proposta por Ridente Júnior et al. (1997), que estabelece pesos para classes de erodibilidade de solos (Quadro 1) e declividades, sendo o mapa final produzido pela multiplicação destes dois planos através de sobreposição (OVERLAY). Índices de erodibilidade de solos do Estado de São Paulo, apresentados por Bertoni \& Lombardi (1985), foram utilizados na elaboração do Quadro 1.

O Quadro 2 apresenta os pesos obtidos pelo cruzamento das classes de erodibilidade de solos (linha) e classes de declividade (coluna).

Considerou-se que áreas suscetíveis à erosão são mais adequadas para recomposição, devido à proteção oferecida pela vegetação ao solo, atenuando os possíveis efeitos causados pela atividade produtiva.

Quadro 1 - Índices de erodibilidade de alguns solos do Estado de São Paulo (Bertoni \& Lombardi, 1985)

Table 1 - Erosivity indices of some soils in the state of São Paulo (Bertoni \& Lombardi, 1985)

\begin{tabular}{|c|c|c|}
\hline Unidade Pedológica & $\begin{array}{c}\text { Índice de } \\
\text { erodibilidade }\end{array}$ & Classe \\
\hline $\begin{array}{l}\text { Cambissolos, solos litólicos. Podzólicos Vermelho Amarelo e Vermelho Escuro } \\
\text { abrúpticos, textura arenosa-média. } \\
\text { Areias quartzosas, relevo suave-ondulado e ondulado. }\end{array}$ & $0,433-0,540$ & I \\
\hline $\begin{array}{l}\text { Podzólico Vermelho-Amarelo não-abrúptico, textura média-argilosa e média. } \\
\text { Podzólico Vermelho-Escuro não-abrúptico, textura média-argilosa. }\end{array}$ & $0,329-0,432$ & II \\
\hline $\begin{array}{l}\text { Podzólico Vermelho-Amarelo e Podzólico Vermelho-Escuro, textura argilosa. } \\
\text { Latossolo Vermelho-Amarelo, textura argilosa. Latossolo Vermelho-Amarelo, } \\
\text { textura média. } \\
\text { Latossolo Vermelho-Escuro, textura média. }\end{array}$ & $0,221-0,328$ & III \\
\hline Latossolo roxo. Terra roxa estruturada. & $0,113-0,220$ & IV \\
\hline $\begin{array}{l}\text { Gleis, Planossolo, Areias quartzosas relevo plano } \\
\text { Solos orgânicos. }\end{array}$ & $0-0,113$ & V \\
\hline
\end{tabular}


- Fertilidade dos solos (FERTILID) - obtida a partir da reclassificação do mapa de solos, considerando-se os dois tipos predominantes: Areias Quartzosas (AQ) consideradas de menor fertilidade, recebendo valor máximo (255), e Latossolos Vermelho-Amarelos (LV2), recebendo valor médio (127). Considerou-se que áreas de menor fertilidade são mais adequadas à recomposição, reservando-se assim, para a atividade produtiva, as áreas de solos com maior fertilidade.

Para o processo de tomada de decisão, adotou-se a metodologia proposta por Eastman et al. (1995) de decisão multicriterial. A preparação dos dados para o processo de decisão iniciou-se pela escolha dos critérios envolvidos, sendo estes comparados dois a dois, segundo uma escala de importância (Quadro 3).

Após a comparação dos critérios, utilizou-se o módulo WEIGHT do IDRISI (Eastman et al., 1995), em que foram obtidos os pesos de cada critério envolvido (escala de 0 a 1 ). Cada critério é representado por um mapa temático, no qual é avaliada sua adequação ao objetivo proposto, em uma escala de 0 a 255 . Considerando $n$ critérios, o mapa de adequação ao objetivo foi obtido por meio do módulo MCE (Multi-Criteria Evaluation) do IDRISI, o qual se baseia na aplicação da seguinte expressão, a cada pixel do mapa:

$$
S=\sum_{i=1}^{n}\left(w_{i} \cdot x_{i} \cdot \Pi c_{j}\right)
$$

em que

$S$ = adequação ao objetivo;

$w_{\mathrm{i}}=$ peso do critério;

$x_{\mathrm{i}}=$ adequação do critério ao objetivo; e

$c_{\mathrm{j}}=$ "score" (0 ou 1) de restrição.

As restrições ao processo de decisão foram as áreas que não poderiam ser alocadas ao objetivo proposto, como estradas, rios, reservas e Áreas de Preservação Permanente já demarcadas. Após a obtenção do mapa de adequação ao objetivo, foi realizado um "ordenamento" das áreas mapeadas por meio do módulo RANK do IDRISI, com a função de organizar as áreas em ordem de adequação.

Para obtenção do mapa de áreas adequadas à recomposição florestal, foram selecionados os melhores 1.000 ha para recomposição, tendo sido demarcadas as regiões que apresentaram maior concentração de pontos. Utilizando o mapa gerado de áreas adequadas à recomposição, foi proposta uma interligação de algumas áreas nativas já existentes, a fim de aumentar a área de Reserva Legal com base nos resultados obtidos.

Quadro 2 - Pesos empregados e suscetibilidade à erosão devido aos fatores solo e declividade, adaptados de Bertoni \& Lombardi (1985)

Table 2 - Weights applied and erosion susceptibility due to the factors soil and slope, adapted from Bertoni \& Lombardi (1985)

\begin{tabular}{|c|c|c|c|c|c|}
\hline $\begin{array}{c}\text { Solos/ } \\
\text { Declividade }\end{array}$ & $\begin{array}{c}\text { Classe V } \\
\text { (1) }\end{array}$ & $\begin{array}{c}\text { Classe IV } \\
\text { (2) }\end{array}$ & $\begin{array}{c}\text { Classe III } \\
\text { ( } 3)\end{array}$ & $\begin{array}{c}\text { Classe II } \\
\text { (4) }\end{array}$ & $\begin{array}{c}\text { Classe I } \\
\text { (5) }\end{array}$ \\
\hline $0-6 \%(1)$ & 1 & 2 & 3 & 4 & 5 \\
\hline De 6 a $12 \%(2)$ & 2 & 4 & 6 & 8 & 10 \\
\hline De 12 a $20 \%$ (3) & 3 & 6 & 9 & 12 & 15 \\
\hline De 20 a $30 \%$ (4) & 4 & 8 & 12 & 16 & 20 \\
\hline$>30 \%(5)$ & 5 & 10 & 15 & 20 & 25 \\
\hline
\end{tabular}

Quadro 3 - Escala de valores e graus de importância entre os dois critérios comparados Table 3 - Value scale and importance degrees between the two criteria compared

\begin{tabular}{|c|c|c|c|c|c|c|c|}
\hline Valores & $1 / 9$ & $1 / 6$ & $1 / 3$ & 1 & 3 & 6 & 9 \\
\hline $\begin{array}{c}\text { Interpretação } \\
\text { (importância) }\end{array}$ & muito menos & menos & pouco menos & igual & pouco mais & mais & muito mais \\
\hline
\end{tabular}


Para efeito comparativo, simulou-se um novo cenário após a interligação, para avaliar as alterações em termos de área e estrutura da paisagem com a nova área de reserva delimitada, em relação ao cenário existente em 1998. As alterações na estrutura da paisagem foram avaliadas por meio dos seguintes índices referentes aos fragmentos de mata nativa: AREA (área em hectares e porcentagem da área total da fazenda), NP (número de fragmentos), PD (densidade, em número de fragmentos/ 100 ha), LPI (índice do maior fragmento, em porcentagem da área total da fazenda), CA (área nuclear, em hectares) e ENN_MN (distância média do fragmento mais próximo, em metros). Os índices utilizados foram calculados por meio do software Fragstats (McGarigal \& Marks, 1995).

\section{RESULTADOS E DISCUSSÃO}

Após a comparação dos fatores dois a dois, obtiveram-se os pesos de importância relativa de cada critério utilizado. O Quadro 4 apresenta os valores de importância utilizados na comparação, bem como os pesos obtidos.

Portanto, o critério mais importante foi a suscetibilidade à erosão (SUSCERO), com peso 0,52, seguido por proximidade à mata nativa (PROXNAT) e proximidade aos rios (PROXRIO), com pesos 0,25 e 0,15, respectivamente. Os demais critérios foram considerados secundários para esta área. Deve-se notar que a proposta da metodologia é oferecer uma alternativa para decisões complexas, envolvendo muitos critérios, e que a seleção dos critérios, bem como a definição dos pesos, deverá variar de acordo com as particularidades de cada área.

A Figura 1 mostra a localização dos melhores 1.000 ha identificados pela metodologia para alocação de áreas de recomposição da mata nativa. Nota-se que neste mapa as áreas estão dispersas, o que inviabilizaria um plano de recomposição. Para facilitar a identificação de possíveis regiões de recomposição, as áreas de maior concentração de pontos foram demarcadas. Neste caso, não foram consideradas as divisões atuais dos talhões, e para implementação destas áreas seria necessário um retalhonamento das áreas produtivas próximas.

Para um melhor resultado, sob a ótica da ecologia de paisagem, seria interessante que as áreas de recomposição ocupassem uma área contínua e extensa, possibilitando maior troca de material genético, trânsito de animais, além de permitir o desenvolvimento de atividades como ecoturismo e educação ambiental (Harris, 1984). No caso desta fazenda, a melhor região identificada, que atende a estes requisitos, seria a área de nascentes localizada na região centro-leste da fazenda. As demais regiões identificadas, apesar de não apresentarem grandes extensões contínuas, estariam interligadas pelas Âreas de Preservação Permanente (APP) em torno dos cursos d'água.

A área escolhida para instalação da nova reserva pode ser observada na Figura 2, juntamente com as Áreas de Preservação Permanente e Reserva Legal já existentes em 1998. A área proposta foi traçada utilizando-se o mapa gerado de áreas adequadas, bem como a distribuição dos fragmentos já existentes. O Quadro 5 mostra algumas diferenças calculadas entre o cenário atual e o simulado, em termos de estrutura da paisagem. A área total de mata nativa (AREA) passaria de 492 ha para 893 ha, totalizando um aumento de cerca de $80 \%$, e as áreas de matas passariam de 7,8 a 14,2\% da área total da fazenda. A criação de um grande fragmento na área proposta estaria elevando o total de área nuclear ${ }^{1 /}$ (TCA), dos 155 ha atuais para 603 ha, ou seja, nesta configuração de reserva a área nuclear seria quadruplicada.

\footnotetext{
1/ Para o cálculo da área nuclear, considerou-se uma borda de $50 \mathrm{~m}$ para efeito de simulação.
}

Quadro 4 - Importância relativa e pesos dos critérios considerados Table 4 -Relative importance and weights of the criteria considered

\begin{tabular}{|l|c|c|c|c|c|c|}
\hline & DECLIV & PROXRIO & PROXNAT & SUSCERO & FERTILID & PESOS \\
\hline DECLIV & 1 & & & & & 0,0359 \\
PROXRIO & 6 & 1 & & & 0,2574 \\
PROXNAT & 6 & $1 / 3$ & 1 & & 0,1530 \\
SUSCERO & 9 & 3 & 6 & 1 & 0,5220 \\
FERTILID & 1 & $1 / 9$ & $1 / 9$ & $1 / 9$ & 1 & 0,0317 \\
\hline
\end{tabular}




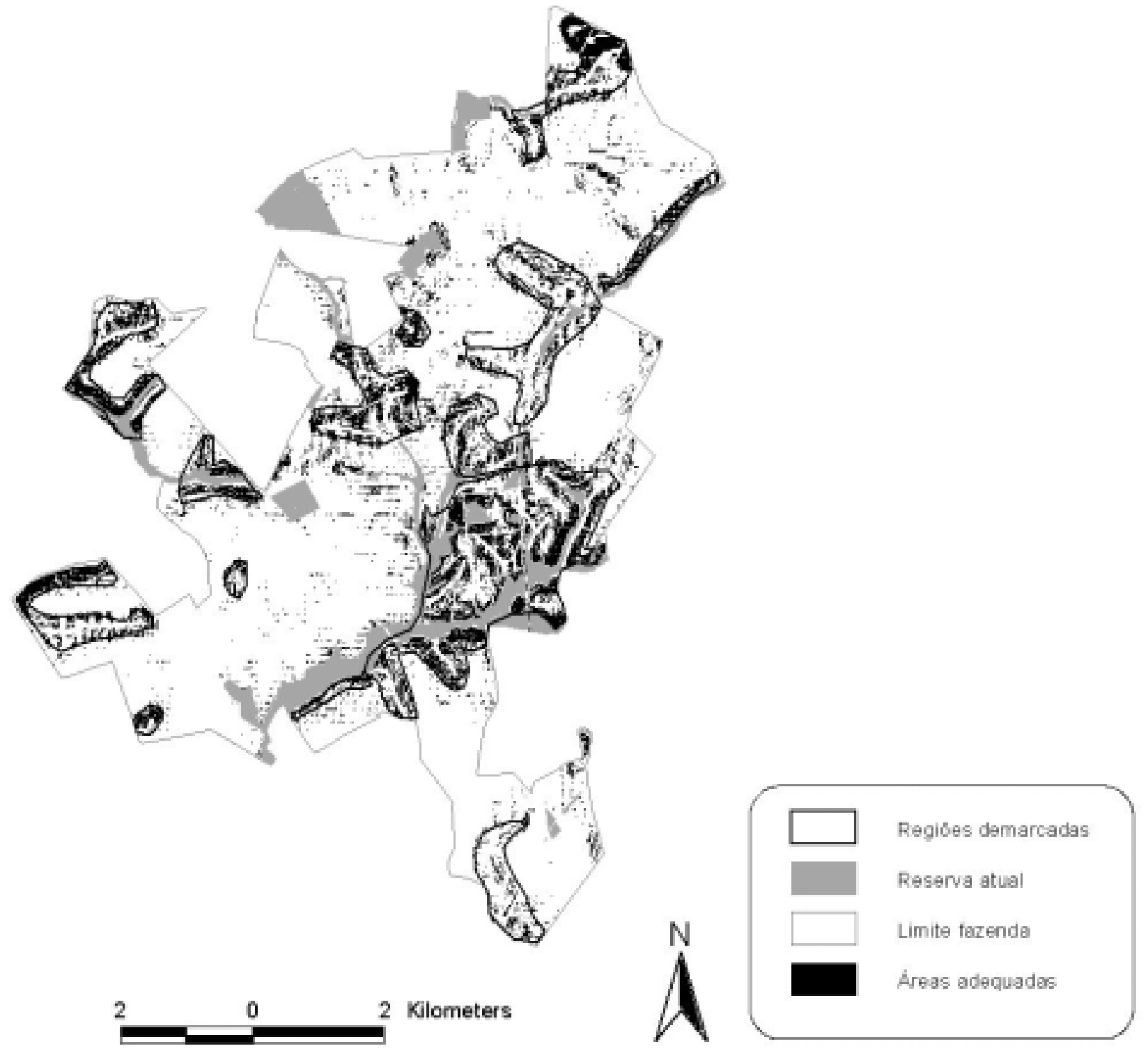

Figura 1 - Proposta de áreas adequadas à recomposição da mata nativa. Figure 1 - Proposal of areas suitable to native forest recovery.

Quadro 5 - Índices calculados para os dois cenários gerados Table 5 - Indices calculated for the two generated scenarios

\begin{tabular}{|l|c|c|c|c|c|c|c|}
\hline \multicolumn{1}{|c|}{ Cenário } & Área (ha) & $\begin{array}{c}\text { Área } \\
(\%)\end{array}$ & $\begin{array}{c}\text { NP } \\
(\mathrm{n})\end{array}$ & $\begin{array}{c}\text { PD } \\
(\mathrm{n} / \mathrm{h} \text { * } 100)\end{array}$ & $\begin{array}{c}\text { LPI } \\
(\%)\end{array}$ & $\begin{array}{c}\text { TCA } \\
(\mathrm{ha})\end{array}$ & $\begin{array}{c}\text { ENN_MN } \\
(\mathrm{m})\end{array}$ \\
\hline Atual & 492 & 7,8 & 39 & 0,16 & 1,71 & 155 & 180 \\
Simulado & 894 & 14,2 & 28 & 0,12 & 8,98 & 603 & 220 \\
\hline
\end{tabular}

R. Árvore, Viçosa-MG, v.27, n.4, p.575-583, 2003 


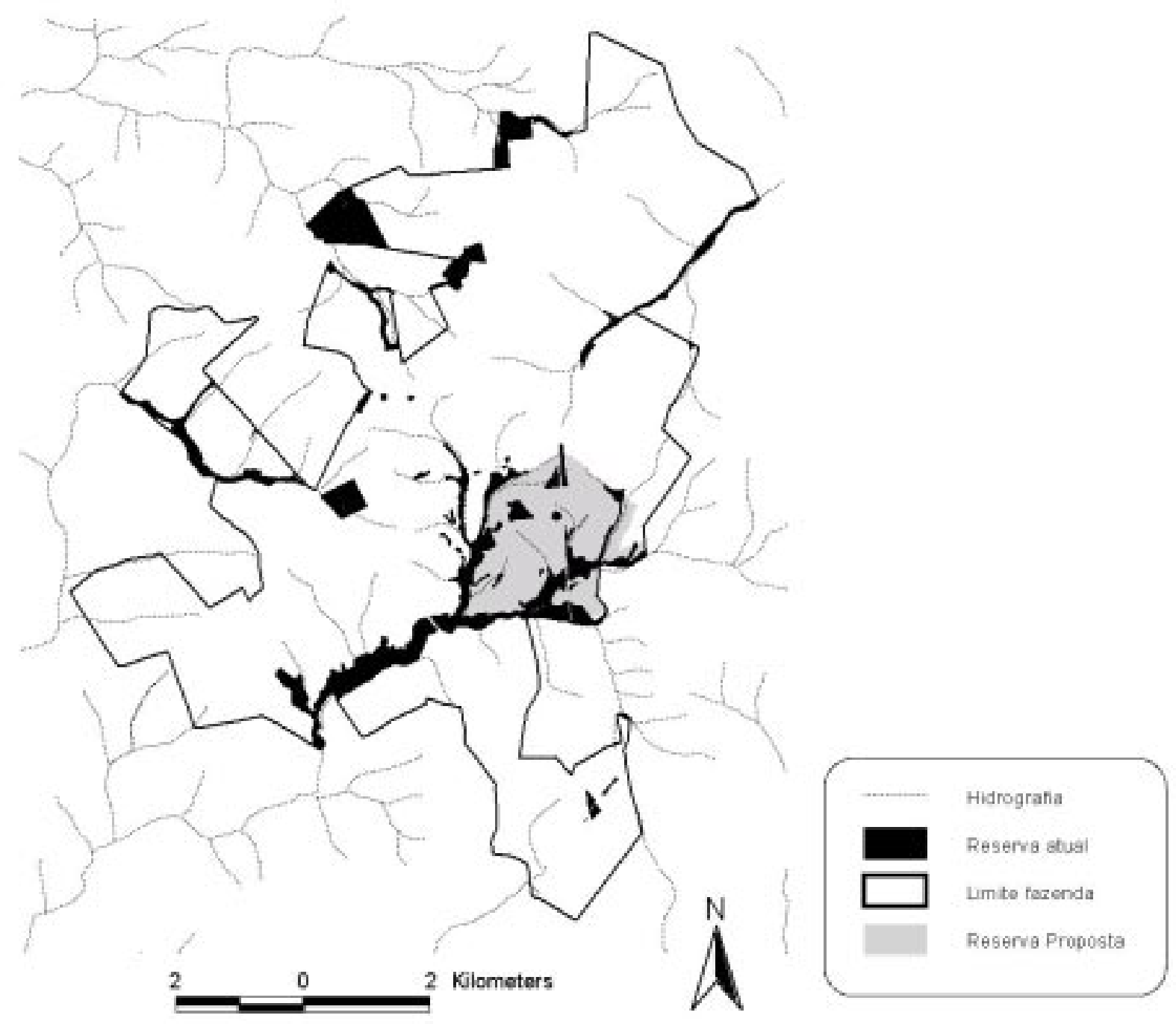

Figura 2 - Áreas de reserva atual e simulada. Figure 2 - Actual and simulated reserve areas.

Ainda, considerando o tamanho dos fragmentos de mata nativa, o índice do maior fragmento (LPI) aumentaria de 1,71 para $8,98 \%$, ou seja, um grande fragmento representaria cerca de $9 \%$ da área total. Com relação à fragmentação da área, a implantação da nova reserva reduziria o número de fragmentos (NP) de 39 para 28 e a densidade de fragmentos (PD), de 0,16 para 0,11 fragmento/100 ha. A distância média dos fragmentos vizinhos (ENN_MN) aumentaria de 180 para $220 \mathrm{~m}$, devido à eventual eliminação de fragmentos próximos, que se localizam na área de instalação da reserva e que seriam interligados, formando um grande fragmento.

\section{CONCLUSÕES}

- O estudo mostrou que a implantação e o manejo de florestas, considerando os aspectos ambientais, sociais, estéticos e legais, segundo os princípios do manejo de paisagens, poderão ser auxiliados por instrumentos como o SIG, no apoio ao processo de tomada de decisões complexas que envolvem muitos fatores espacialmente distribuídos;

- a metodologia utilizada no processo de tomada de decisão (análise multicriterial) facilitou a atribuição de pesos aos fatores empregados, com a utilização do 
método de comparação pareada. Além disto, o resultado final pode expressar todos os fatores integrados de forma ponderada. O mapa de adequação à recomposição florestal poderá ser útil no auxílio ao planejamento da alocação de novas áreas para composição da Reserva Legal; e

- a simulação de um novo cenário permitiu a avaliação das alterações na estrutura da paisagem em termos de tamanho e forma dos fragmentos de mata nativa, caso a área fosse implementada, mostrando que este tipo de análise poderá ser útil na avaliação final de um projeto de recomposição.

\section{AGRADECIMENTO}

Aos professores Dr. Hilton Thadeu Zarate do Couto, Dr. José Luiz Stappe e Dr. Fernando Seixas, do Departamento de Ciências Florestais da ESALQ/USP, pelo apoio, pelas sugestões e pelo auxílio na determinação dos pesos.

À direção e aos funcionários da empresa International Paper do Brasil Ltda., especialmente aos engenheiros Denilson de Jesus, pelo fornecimento de todas as informações necessárias e apoio ao trabalho, e ao Fernando A. C. Bilia, pelo apoio e pela disponibilização da fazenda "Santa Fé B" como área de estudo.

À FAPESP, pelo apoio financeiro ao desenvolvimento do projeto (procs. 00/3740-5; 01/14172-0).

\section{REFERÊNCIAS BIBLIOGRÁFICAS}

BERGEN, S. D. et al. Predicting the visual effect of forest operations. Journal of Forestry, v. 93, n. 2, p. 26-28, 1995.

BERTONI, J.; LOMBARDI NETO, F. Conservação do solo. Piracicaba: Livroceres, 1985. 392 p.

BOYCE, S. G.; McNAB, W. H. Management of forested landscapes - simulations of three alternatives. Journal of Forestry, v. 92, n. 1, p. 27-32, 1994.

EASTMAN, J. R. et al. Raster procedures for multi-criteria/ multi-objective decisions. Photogrammetric Engineering \& Remote Sensing, v. 61, n. 5, p. 539-547, 1995.

FERRAZ, S. F. B.; VETTORAZZI, C. A. Mapeamento de risco de incêndios florestais por meio de um sistema de informações geográficas. Scientia Forestalis, n. 53, p. 3948, 1998.

R. Árvore, Viçosa-MG, v.27, n.4, p.575-583, 2003
FORMAGGIO, A. R.; ALVES, D. S.; EPIPHANIO, J. C. N. Sistemas de Informações Geográficas na obtenção de mapas de aptidão agrícola e de taxa de adequação de uso das terras. Revista Brasileira de Ciência do Solo, v. 16, p. 249-256, 1992.

HARRIS, L. D. The fragmented forest. Chicago, The University of Chicago Press, 1984. 211 p.

LACHOWSKI, H. M. et al. Remote Sensing and GIS: their role in ecosystem management. Journal of Forestry, v. 92, n. 8, p. 39-40, 1994.

LATHROP JR., R. G.; BOGNAR, J. A. Applying GIS and landscape ecological principles to evaluate land conservation alternatives. Landscape and Urban Planning, v. 41, p. 27-41, 1998.

MAGRO, T. C. Manejo de paisagens em áreas florestadas. Silvicultura, v. 18 , n. 69, p. 38-45, 1997.

OLIVEIRA, J. B.; ALMEIDA, C. L. F.; PRADO, H. Levantamento Pedológico Semidetalhado do Estado de São Paulo - Quadrícula de Brotas. Convênio Embrapa Secretaria de Agricultura e Abastecimento do Estado de São Paulo. Escala 1:100.000. 1981.

OLIVER, C. D. A landscape approach: achieving and maintaining biodiversity and economic productivity. Journal of Forestry, v. 90, n. 9, p. 20-25, 1992.

McGARIGAL, K.; MARKS, B. J. Fragstats: spatial pattern analysis program for quantifying landscape structure. Gen. Tech. Rep. PNW-GTR-351. Portland, OR: U.S. Department of Agriculture, Forest Service, Pacific Northwest Research Station, 1995. 122 p.

RIDENTE JÚNIOR, J. L. et al. Mapa de risco potencial à erosão em área piloto do município de São José do Rio Preto-SP, utilizando Sistema de Informação Geográfica. In: SIMPÓSIO DE USUÁRIOS IDRISI, 2., 1997, Campinas. Anais... Campinas: 1997. p. 36-37.

RODRIGUES, L. C. Relação entre parâmetros silviculturais e concentração de P, K, Ca e Mg em árvores de Eucalyptus saligna Smith, em Brotas-SP. 1986. 56 f. Dissertação (Mestrado em Ciências Florestais) - Escola Superior de Agricultura "Luiz de Queiroz", 1986.

VETTORAZZI, C. A.; VALENTE, R. O. A.; BALLESTER, M. V. R. Forest fire hazard mapping in a GIS environment for a river basin in the State of São Paulo, Brazil. In: INTERNATIONAL CONFERENCE ON GEOSPATIAL INFORMATION IN AGRICULTURE AND FORESTRY. 2., 2000, Lake Buena Vista. Proceedings... Ann Arbor: ERIN International, MI, 2000. p. 609-616. 
VIANA, V. M.; OLIVEIRA, R. E. Manejo de paisagem e a sustentabilidade de plantações florestais. In: WORKSHOP SOBRE MANEJO DE PAISAGENS EM ÁREAS FLORESTADAS, 1997, Piracicaba. Anais... Piracicaba: 1997. p. 23-45.
ZHOU, J.; CIVCO, D. L. Using genetic learning neural networks for spatial decision making in GIS.

Photogrammetric Engineering \& Remote Sensing, v. 62, n. 11 , p. $1287-1295,1996$. 